\title{
La neuroéducation : mieux comprendre le cerveau pour mieux enseigner
}

https://doi.org/10.24046/neuroed.20120101.3

\author{
Steve MASSON ${ }^{1}{ }^{*}$
}

1 Université du Québec à Montréal

*Courriel : masson.steve@uqam.ca

Depuis une dizaine d'années, une nouvelle approche de recherche se développe à un rythme exponentiel : la neuroéducation. Cette approche jette un regard inédit sur les problèmes éducatifs rencontrés par les élèves et les enseignants, en examinant ces problèmes à un niveau d'analyse jusqu'ici inexploré : le niveau cérébral. À l'aide de l'imagerie cérébrale, la neuroéducation cherche notamment à identifier les mécanismes cérébraux liés aux apprentissages scolaires et à l'enseignement, mais aussi à mieux comprendre comment la connaissance de ces mécanismes peut contribuer à l'amélioration des pratiques enseignantes.

La revue Neuroéducation a été créée dans le but de faciliter le développement de ce nouveau champ de recherche en mettant à la disposition des chercheurs en neuroéducation un lieu d'évaluation et de partage des connaissances, c'est-à-dire un lieu où il est possible de soumettre des données, des méthodes et des idées nouvelles au regard des pairs. Pour cette raison, vous trouverez dans cette revue des articles présentant non seulement des recherches expérimentales, mais aussi des recherches expérimentales en préparation, des recherches théoriques proposant des hypothèses et de nouveaux modèles d'apprentissage et d'enseignement, des discussions méthodologiques, des revues de la littérature, des commentaires et des résumés de livres.

Ce premier numéro regroupe cinq articles. Trois présentent des résultats de recherche: le premier porte sur des mesures électroencéphalographiques acquises en contexte authentique et les deux autres portent sur le 
développement de la capacité d'inhibition chez les enfants. Un quatrième article propose un modèle pour l'enseignement des langues secondes et étrangères s'appuyant sur des études en neuroimagerie et le cinquième est un commentaire portant sur l'intérêt d'utiliser des mesures psychophysiologiques pour mieux comprendre l'apprentissage.

En terminant, il convient de souligner le travail remarquable des assistants à la rédaction (Geneviève Allaire-Duquette, Lorie-Marlène Brault Foisy, Jan-Sébastien Dion, Stéphanie Lafortune, François Thibault et Élaine Turmel) et des professeurs-chercheurs ayant contribué à l'évaluation des articles. Sans le travail dévoué de ces personnes, la publication de cette revue ne serait pas possible. 